\title{
recherche participative : contribution à partir d'un travail effectué avec des migrants dans la ville de Coimbra ${ }^{1}$
}

\author{
Elsa Lechner ${ }^{2}$
}

\section{Résumé}

L'article a pour objet de présenter les fondements théoriques et les enjeux sociaux et politiques de la recherche participative à travers la pratique concrète d'une recherche conduite dans le contexte migratoire de la ville de Coimbra. Menés à la lumière des concepts fondamentaux de l'investigation-action (I-A), la description des modalités et l'analyse des résultats de la recherche en cours mettent en évidence la dimension de production collective des savoirs liée à la démarche participative et la contribution qu'elle peut représenter à la construction d'un monde commun.

\section{Abstract}

Based on an ongoing project of participatory research with immigrants in Coimbra (Portugal), this paper presents the theoretical underpinnings and the political challenges faced by this type of research. Focusing on the key concepts of action research, we discuss the modalities and preliminary results of our research project while highlighting the collective dimension of

\footnotetext{
${ }^{1}$ Ce texte a été écrit à l'occasion du $\mathrm{X}^{\text {ème }}$ Séminaire international «Images de la culture, culture des images », qui a eu lieu à l'École de la communication et des arts de l'Université de São Paulo (Brésil) du 28 au 30 août 2013. Il s'inscrit dans le cadre d'un projet de recherche en développement, coordonné par l'auteure, mené au Centre d'Etudes Sociales de l'Université de Coimbra (PTDC/CS-ANT/111721/2009).

${ }^{2}$ Elsa Lechner est anthropologue, chercheure et directrice de projet au Centre d'Etudes Sociales de l'Université de Coimbra (Portugal).
} 
knowledge production in collaborative research. The paper also reflects on the potential contribution of participatory research to the construction of a "common world".

MOTS CLES : recherche participative, épistémologie civique, production collective de savoirs.

KEYWORDS: Participatory research, civic epistemology, collective knowledge production.

Ce texte vise à définir la notion de recherche participative selon une lecture ancrée, simultanément, dans la généalogie théorique de la littérature scientifique de la spécialité et dans la pratique concrète d'une recherche en collaboration menée dans le contexte portugais.

Pour ce faire, nous avons revisité les auteurs de référence, créateurs du terme, et leurs disciples, héritiers de la recherche-action, en attestant de la parfaite actualité de leurs présupposés et de leurs objectifs communs de travail.

Nous présentons ensuite la recherche en cours au Centre d'Etudes Sociales de l'Université de Coimbra, où notre équipe pluridisciplinaire de recherche met en pratique des ateliers de travail biographique avec le concours d'immigrants selon une méthode participative ${ }^{3}$.

Nous montrons comment la recherche participative est un défi tant théorique que pratique et civique. Mais nous constatons aussi que l'expérience participative, par l'audace de la démarche égalitaire qu'elle propose dans un monde d'inégalités, permet de poser les bases d'un bien-être commun utile à toutes les parties: institutions académiques, recherche scientifique, immigrants, institutions publiques, sens commun (représentations, communication, interconnaissance).

\section{Concepts et applications : la collaboration en tant que participation active dans la transformation sociale}

\footnotetext{
${ }^{3}$ Les ateliers biographiques ont fait l'objet d'un article publié dans la revue brésilienne Educação \& Realidade (Lechner, 2012).
} 
Le terme « recherche participative » désigne, de manière globale, un ensemble de processus de recherche qui ont recours à des méthodologies d'investigation activement liées aux communautés et/ou aux décideurs des politiques publiques. Une recherche participative, selon le Center for Collaborative Research de l'Université de Californie, implique trois éléments essentiels : 1) elle doit être orientée vers l'équité, 2) réalisée selon des formats de collaboration, 3) effectuée avec le concours des communautés.

Cette définition soulève plusieurs questions qui méritent d'être précisées, ne serait-ce que pour définir ce que l'on entend par «collaboration » et par «communauté » dans un contexte de recherche académique et/ou scientifique. Mais, avant de se lancer dans ce développement conceptuel, il est important de présenter la généalogie théorique et pratique de ce type de recherche, et les liens qu'elle entretient avec l'investigation-action et à la recherche partagée.

L’investigation-action (I-A) a pour objet de résoudre des problèmes immédiats ou de mener à terme un processus de réflexion sur la résolution progressive d'un problème social. Elle est conduite par des équipes pluridisciplinaires, unies par leur appartenance à une « communauté d'usages » et qui visent à améliorer la compréhension et la résolution de questions précises affectant la vie des personnes et des institutions. Elle est pragmatique et auto-réflexive.

La démarche de l'investigation-action a été conçue aux États-Unis, dans les années 1940, par Kurt Lewin, un psychologue social, spécialiste des relations et du groupe, dans la continuité de la pensée de John Dewey (1938) sur l'éducation par l'expérience. Dans un texte inaugural datant de 1946, intitulé «Action Research and Minority Problems », Lewin décrit l'I-A comme une recherche visant à transformer une situation ou un problème et se développant selon des phases ou des étapes en spirale, chaque étape étant composée d'un cycle successif - prévision, action, découverte - en relation avec la progression et la résolution du processus de transformation.

Pendant longtemps, l'I-A n'a pas été bien reçue par les sciences sociales car elle allait à l'encontre de leur souci de scientificité et de légitimation, à une période d'expansion du néolibéralisme économique et d'obsession quantitative dans le domaine scientifique. Ce n'est que plus récemment, avec le besoin d'associer la recherche à la résolution pratique de problèmes sociaux - dans des domaines comme l'habitation, la santé, le travail - que l'I-A a pu être reconnue et développée, plus particulièrement dans les pays nordiques. De nos jours, comme l'affirme Wilfred Carr (2013), l'I-A participe à l'élaboration même des formes de vie démocratique qu'elle vise à créer et dont elle constitue un élément essentiel. 
Au Brésil, les chercheurs Sergio Bairon et de Caio Lazaneo utilisent le terme de « recherche partagée » dans le cadre de leurs travaux en anthropologie visuelle (Bairon \& Lazaneo, 2012). La recherche partagée est l'héritière directe de l'Education Populaire de Paulo Freire (1987) et elle est proche de la recherche participative, telle qu'elle est comprise dans le monde anglo-saxon (Chevalier \& Buckles, 2013). Les praticiens de la recherche partagée intègrent et combinent dans leur travail trois aspects fondamentaux : 1) la participation de tous ceux qui sont concernés par la recherche, la société et la démocratie, 2) l'action engagée par l'expérience et l'histoire, 3) la poursuite d'une avancée de la pensée et de la connaissance. Le dialogue et la dimension collective de la démarche sont les principes de base de la recherche partagée, en tant que facteurs de développement aussi bien local que global. L'enjeu en est la mise en pratique de la délibération collective et de la participation démocratique dans la sphère de la connaissance à tous les niveaux de notre monde «global», depuis la vie au sein d'une communauté d'indiens d'Amazonie jusqu'au fonctionnement d'organisations régionales, nationales et internationales (Chevalier \& Buckles, 2013).

Selon Chevalier et Buckles, les questions sociales doivent être abordées « socialement », conjointement avec l'ensemble des parties intéressées, et non pas uniquement en fonction d'intérêts privés ou du point de vue de spécialistes. Les éléments qui ressortent de ces délibérations collectives doivent être totalement intégrées à des processus partagés de production de la connaissance, de planification et de prise de décision. La connaissance dont il est ici question est une connaissance vivante, impliquant la participation des acteurs sociaux aux analyses approfondies de leurs propres situations de vie, et capable de créer le «bien commun » dans l'acception la plus large possible d'un « bien-monde ». Ces auteurs proposent ainsi une notion élargie de la «communauté » qui englobe le bien commun, à construire et à partager par tous.

En vérité, on trouve déjà cette notion élargie de communauté dans le texte initial de Kurt Lewin (1946), lorsqu'il indique que le problème des minorités est aussi celui des majorités : «Le problème des noirs est le problème des blancs, tout comme le problème des juifs est celui des non-juifs » (Lewin, 1946, p. 44). Et, c'est dans la même logique d'intégration globale que l'idée de collaboration est elle aussi explicitée par cet auteur, lorsqu'il suggère de penser la recherche et ses problèmes non pas en termes généralistes mais en termes existentiels, à partir de l'expérience concrète de celles et ceux qui y participent. "Coopération» est le mot qu'emploie l'auteur, à l'horizon d'une « gestion sociale visant la pratique ». Quand, où et par qui doit être faite la recherche, telles sont les questions auxquelles il faut répondre, en partant 
du principe que l'on doit adopter une attitude sociale de « regard et d'écoute sur les processus même de l'action ».

Cette précision généalogique concernant les concepts de communauté et de collaboration est importante, non seulement pour rendre justice à la vision de Lewin, mais également parce que, sous la forme élargie et intégrative dont celui-ci conçoit la recherche participative, elle permet d'éclairer le triangle dynamique formé par la recherche, l'action et la formation et la manière dont leur association participe à l'édification d'un «bien commun ».

Le travail collectif (en tant que méthodologie) et les relations inter-groupales (en tant que sujet d'étude) étaient, pour ce professeur du Massachussets Institute of Technology, l'un des aspects les plus essentiels des «scènes nationales et internationales ». On peut retrouver dans ces scènes, ou dans ces paysages, une dimension politique de sa lecture des phénomènes sociaux et de ses propositions de recherche-action-formation. Le bien-fondé du travail avec et pour des groupes tel qu'il l'a expérimenté et mis en œuvre dans la période post deuxième guerre mondiale semble être toujours d'actualité. Il est presque effrayant de constater que ce que Lewin écrit en 1946 reste vrai de nos jours : "Nous savons, aujourd'hui plus que jamais, qu'elles [les relations inter groupe] sont de la dynamite en puissance. La stratégie de recherche sociale doit prendre en considération les dangers qui leur sont inhérents. » (Lewin, 1946, p. 44). Près de soixante-dix ans après, les conflits entre groupes sociaux et entre pays restent les mêmes, les préjugés et la lutte pour le pouvoir continuent à fournir les ingrédients de la discorde, comme si l'histoire n'avait pas fourni suffisamment d'exemples criants des conséquences néfastes de ces conflits. Les leçons de Lewin formulées dans les années 1940 gardent ainsi toute leur pertinence et leur actualité quant au besoin de comprendre les dynamiques sociales et de faire de la recherche sociale dans une logique collective, de dialogue, participative, visant à la construction du bien commun.

Les domaines sociaux, éducatifs et de la santé sont ceux qui ont le plus souvent été investis par l'investigation-action ainsi que par la recherche participative et partagée. Soulignons également le rôle des arts, notamment du théâtre, dans cet effort de compréhension des réalités sociales, au travers du travail de groupe, du jeu de rôles, et des actions d'innovation ou de transformation sociale qui en découlent. Les démarches du «théâtre de l'opprimé » d'Augusto Boal (1971) sont l'exemple le plus connu de ces pratiques, même si de nombreuses autres ont suivi. Les objectifs en sont la démocratisation des moyens de production théâtrale, l'accès des couches sociales les moins favorisées à ce moyen d'expression et la transformation 
de la réalité par le dialogue et l'expression théâtrale ${ }^{4}$. Il en va de même pour certaines démarches menées dans le domaine de $1^{1}$ architecture $^{5}$, qui impliquent la participation des acteurs directement concernés et poursuivent avec eux les mêmes objectifs de production de connaissance ${ }^{6}$.

Longtemps, dans les sciences sociales, l'Investigation-Action et la recherche collaborative/participative ont été le parent pauvre de l'activité et de la production scientifiques, comme l'affirme José Ferreira de Almeida (2001). Cependant, le même auteur mentionne également un changement significatif de cette tendance à partir des années 19801990, grâce au travail des anthropologues et des sociologues sur des terrains sociaux tels que l'habitat, la lutte contre la pauvreté, la santé, et au sein d'institutions autres que l'université. Un autre aspect auquel Ferreira de Almeida fait référence est l'encadrement institutionnel du développement de la recherche scientifique elle-même, progressivement marqué par les principes et les objectifs du «développement technologique et de l'innovation ». Dans ce contexte, le terme «innovation» semble être la version technocratique de l'idée de transformation sociale. Innover, tout comme transformer, c'est apporter quelque chose de nouveau. Néanmoins, la connotation politique du mot «innovation » est moins forte et moins chargée d'utopie que l'idée de transformation. Sous des tendances et des styles qui varient en fonction des idéologies et des groupes de pouvoir auxquels les agents et acteurs de chacun de ces programmes - transformation ou innovation - associent leurs actions, on peut toutefois trouver une visée commune de changement social.

En effet, aussi bien au sein de l'Union Européenne qu'aux États-Unis ou en Australie, les politiques scientifiques dessinent au présent des horizons marqués par ces grandes lignes. Remarquons dans ce sens que le financement de projets de recherche soucieux d'agir directement sur les réalités sociales a augmenté. À titre d'exemple, rappelons la création en 2009-2010 du Collaborative Research Network (CRN) en Australie qui a pu compter, rien que pour les deux premières années de son existence, sur l'attribution de 81 millions de dollars

\footnotetext{
${ }^{4}$ La Charte de l'Association Internationale du Théâtre de l'Opprimé (OITO) fixe l'objectif, dans le premier point de son préambule, d' « humaniser l'humanité ».

${ }^{5}$ Cf. Bouman O. (2008). "Unsolicited Architecture". A collaborative project by Archis + AMO + C-LAB, Studio for Unsolicited Architecture, Design, and E-Culture, the Netherlands Architecture Institute.

${ }^{6}$ Dans le cadre de notre projet de recherche participative au CES, nous nous proposons d'ouvrir une formation, co-organisée avec les collègues du département d'architecture de l'Université de Coimbra, dans laquelle nous ferons un exercice suivi de dialogue et de partage interdisciplinaires autour d'un Centre commercial. Actuellement déserté par les habitants de Coimbra, cet espace a été plusieurs fois cité par les immigrants volontaires du projet comme un lieu de référence urbaine dans leurs vies de citadins (Lechner \& Paulo Providência, 2013).
} 
pour la mise en route de 15 projets $^{7}$. Le programme d'innovation pour le XXI ${ }^{\mathrm{e}}$ siècle, dans lequel s'inscrit le CRN, souligne l'importance du rôle qu'a tenu la collaboration dans la promotion de la recherche de pointe en Australie et dans le monde entier. Il semblerait que les gouvernements commencent, à présent, à pratiquer ce qui avait déjà été suggéré par Lewin dans les années 1940.

Mais les enjeux restent les mêmes. La collaboration exige des partenariats égalitaires dans des contextes sociaux marqués par l'asymétrie. Elle requiert le partage du pouvoir, des ressources, des crédits, des résultats, des connaissances ainsi que l'estime réciproque du savoir et de la spécificité des partenaires entre eux. Il s'agit d'un processus interactif qui conjugue la recherche, la réflexion et l'action et amène à leur progression conjointe. C'est dans le cadre de tels enjeux que nous présenterons les défis concrets que nous avons dû relever au sein de notre projet d'investigation.

\section{Une intention en construction : les défis du contact, de la relation, de la communication et de la co-production}

Le projet «Recherche des migrations et approche biographique : construire un travail en collaboration dans le contexte portugais », financé par la Fondation pour la Science et la Technologie, est une proposition de travail en collaboration entre des chercheurs en sciences sociales et des immigrants habitant au Portugal. Plus particulièrement, nous cherchons à étudier et à connaître l'expérience migratoire d'immigrants aux origines (onze pays différents) et profils très diversifiés (hommes, femmes, travailleurs, étudiants, chômeurs, jeunes, moins jeunes, lettrés et illettrés) et qui ont choisi - d'une façon ou d'une autre - la ville de Coimbra comme lieu de vie.

Les objectifs de départ de ce travail découlent de notre intérêt majeur pour les expériences et les récits biographiques des sujets qui sont nos interlocuteurs ${ }^{8}$. Les questions que nous souhaitons explorer sont les suivantes : 1) quel est le point de vue des migrants sur leur expérience migratoire ? 2) Quel est l'impact de la recherche dans la vie des migrants et dans les sociétés d'immigration? 3) Comment faire pour que les personnes que nous rencontrons ne soient pas des «objets de recherche» mais soient en position de sujets partenaires de la recherche?

\footnotetext{
7 www.innovation.gov.au/CRN

8 Nous utilisons le masculin universel en y incluant toutes nos interlocutrices, femmes et amies, volontaires pour le projet.
} 
Pour mener cette enquête, nous avons investi le terrain de la ville de Coimbra, tout en faisant une analyse de la législation portugaise sur l'immigration et des discours médiatiques tenus à ce sujet au Portugal. Cette confrontation entre le discours normatif sur l'immigration et les expériences vécues par des migrants «en chair et en os » est un point essentiel de notre travail analytique, de notre proposition méthodologique basée sur des ateliers biographiques ainsi que de notre effort de sensibilisation et de diffusion des résultats à travers des productions multimédias (film documentaire, DVD).

En ce sens, bien que nous ayons rencontré de nombreux obstacles dans la recherche de volontaires au cours de la première année (difficulté de la prise de contact, question de l'affinité des motivations pour entreprendre la collaboration), les activités réalisées en 2011 ont néanmoins lancé les bases d'un travail d'épistémologie civique (Jasanoff, 2004a, 2004b) et de théorie appliquée qui, depuis, a entraîné d'autres défis, fort enrichissants, tant pour notre recherche que pour notre réflexion, sur les formats participatifs, sur l'étude des migrations, et ce, grâce aux migrants eux-mêmes. Par la suite, les activités développées durant l'année 2012 ont fait germer ces premières tentatives, en créant des occasions de rencontre avec les communautés de migrants, les institutions publiques et les services de l'État qui ont entrainé un engagement croissant de l'équipe de recherche, des volontaires et des institutions locales.

Durant cette deuxième année de vie du projet, le travail sur le terrain a été de contacter les institutions et les personnes susceptibles de nous faciliter l'accès à des volontaires potentiels pour les ateliers biographiques. Nous avons également mis en place des rencontres et des interviews individuelles avec des participants. En janvier 2012, nous avons tenu une première réunion avec le Réseau Social de la Mairie de Coimbra (Câmara Municipal de Coimbra-CMC). Cette rencontre a permis de faire connaître le projet et de recevoir une aide officielle dans la recherche de volontaires. En effet, à la suite de cette première rencontre, d'autres réunions ont été programmées entre l'équipe de recherche et les personnels de l'action sociale de la CMC qui nous ont mis en contact, par le biais notamment des Services de l'habitat et de la famille, avec des migrants habitant la ville. Le 30 janvier 2012, nous avons rencontré le Service de l'habitat de la Mairie du quartier d'Ingote, rencontre qui nous a valu de recruter quelques volontaires pour les ateliers. D'autres l'avaient été dans le milieu universitaire, où un large pourcentage d'étudiants étrangers, ayant le statut légal d'immigrants au Portugal, se sont montrés intéressés et ouverts à une participation. Au total, nous avons contacté près de 100 personnes, parmi lesquelles nous avons maintenu un lien régulier avec 20 d'entre elles. Le rôle des institutions et des médiateurs a été décisif tout au long de cette étape de construction du travail en collaboration. 
En juin 2012, nous avons fait une présentation publique du projet, à l'occasion du séminaire «Ruminations urbaines ${ }^{9}$ : racontez votre ville dans une ronde d'histoires » à la Maison de la Culture. Quelques-uns des immigrants que nous avions contactés au préalable y ont participé, ainsi que le responsable du Service de l'Education et de la Culture de la mairie. Ce n'est qu'à la suite de ces contacts que nous avons pu organiser deux ateliers biographiques (en juillet et en octobre 2012). Seuls des étudiants (originaires de cinq pays : Argentine, Brésil, Cap Vert, Chine et Ukraine) ont participé au premier; pour le second, nous avons eu la présence d'un groupe mixte, plutôt hétérogène (étudiants, non-étudiants, un immigrant illettré) issu de sept pays différents : Angola, Australie, Brésil, Cap Vert, Côte d'Ivoire, São Tomé, Ouzbékistan. Chacun de ces ateliers a duré trois jours et s'est déroulé selon les mêmes structure et protocole d'exercices (écoute, partage, résonances) analysés dans l'article déjà référencé (Lechner, 2012).

La mise en place de ces deux ateliers biographiques a permis d'identifier les grands thèmes à approfondir pour l'analyse du projet. Il s'agit de la discrimination qui peut prendre plusieurs faces lorsqu'il s'agit de femmes immigrantes noires et de brésiliennes célibataires ou divorcées), du racisme - notamment de la part des services de l'État directement dédiés aux immigrants (c'est le cas du Services des Etrangers et des Frontières dans leur accueil du public) - et de l'importance des églises et des religions pour ce qui est de l'accueil et des réseaux de solidarité consacrés aux immigrants. Il ressort de ces ateliers que beaucoup de stéréotypes, de préjugés et finalement d'aveuglement règne dans le rapport aux immigrants. Ce contre quoi les ateliers biographiques viennent justement apporter leur contribution grâce à la circularité et l'horizontalité des échanges qu'ils suscitent et à leurs effets formateurs et transformateurs. En juin dernier, nous avons réalisé un seul atelier consacré aux religions, auquel ont participé six représentants de confessions diverses (baptiste, catholique, spirite, messianique, mormone, musulmane) et au cours duquel les participants ont exprimé leur étonnement devant le respect mutuel manifesté entre personnes de différentes croyances.

Il ne fait aucun doute que le travail de contact avec les volontaires du projet et les participants aux ateliers biographiques a relevé le défi de créer une collaboration dans des contextes sociaux asymétriques. Et c'est justement parce que cette différence structurelle entre le monde de l'université et les réalités du monde de l'immigration (moins évidente certes durant le premier atelier d'étudiants) est devenue palpable dans notre expérience de recherche

\footnotetext{
${ }^{9}$ Ce titre joue sur les résonances en portugais du mot « rumination » et sur le double sens qu'on peut y entendre en relation avec les récits de la ronde : «ruminer » (remâcher, ressasser, avoir sans cesse à l'esprit) et « agir » sur le thème ou le domaine de la discussion, dans ce cas précis, la ville.
} 
que nous avons organisé les troisième et quatrième ateliers, directement centrés sur la question des femmes ${ }^{10}$ (mars 2013) et des religions (juin 2013). Durant ces ateliers, nous avons pu connaître d'autres expériences qui ont approfondi l'ancrage de notre travail participatif dans les réalités migratoires. Nous avons appris, par exemple, l'existence d'une mosquée dans la ville de Coimbra, inconnue notamment du Réseau Social de la Mairie. C'est bien grâce à notre projet que les personnels de ce service de la CMC en ont eu connaissance.

Nous comprenons ainsi comment la participation dans des contextes d'asymétrie sociale ou statutaire (entre nationaux/étrangers ; universitaires/illettrés, etc.) mène à des « réciprocités asymétriques » (Temple, 2003) qui apportent, au cœur du processus de travail participatif avec des immigrants, une dimension d'altérité qui potentialise l'apprentissage mutuel et ouvre un espace de transformation par le dialogue, l'un et l'autre pouvant conduire à une action novatrice dans la sphère collective. Encore faut-il que les partenaires engagés dans cette relation d'apprentissage et de dialogue aient le souhait d'agir en ce sens, de façon constructive, au-delà des statuts sociaux de départ. Comme le dirait Temple, il faut que la valeur symbolique de l'échange ne se superpose pas à la valeur réelle du partage lui-même. Ou encore, il faut que les valeurs symboliques associées aux différents statuts sociaux de départ ne bloquent pas, par leur propre poids dans la production partagée, la possibilité de transformation des valeurs que sont la responsabilité et la production commune. Et nous trouvons là encore de nouveaux défis, notamment ceux de la relation et de la communication entre des personnes de culture, de langue et de langages différents.

Or, les outils les plus utiles au «travail intérieur » inhérent à ces défis théoriques, méthodologiques et civiques de la collaboration sont l'enregistrement et l'analyse des images du processus de travail lui-même. C'est pourquoi nous avons filmé et enregistré la totalité des heures d'atelier biographique (60 heures), avec l'autorisation de tous les participants.

$\mathrm{Au}$ début, un seul participant n'a pas souhaité être filmé. Mais, c’est justement après qu'il ait livré son témoignage (il fut l'un des premiers à le faire) sur la discrimination et le racisme vécus au comptoir d'accueil du SEF - « Loja do Cidadão ${ }^{11}$ qu'il a lui-même reconnu l'importance de faire connaître son expérience à un public plus large et anonyme. Nous disposons ainsi d'un corpus audiovisuel très riche qui fait l'objet d'analyses classées par thèmes et qui sera intégré au DVD consacré au projet et au film documentaire que nous réaliserons sur le thème de l'immigration.

\footnotetext{
10 Atelier qui a eu lieu au Centre d'accueil Jean Paul II avec un groupe de femmes issues du Brésil, de la GuinéeBissau, du Mozambique et d'Ukraine, bénéficiaires des aides sociales du Centre.

${ }^{11}$ Pôles d'accueil destinés au traitement de documents administratifs.
} 


\section{Un peu d'analyse : la construction participative du Centre commercial Avenida en tant que thème (involontaire) du projet}

Durant le deuxième atelier biographique, mis en place avec un groupe mixte en octobre 2012, le thème du Centre commercial Avenida (CCA) est apparu de façon inopinée. Il s'agit d'un Shopping Center construit dans les années 1980 sur l'une des principales avenues de Coimbra (l'avenue Sá da Bandeira), à l'emplacement d'un ancien théâtre du XIX siècle, le seul où l'on pouvait encore écouter de l'opéra en ville. Objet de polémiques, le CCA a été érigé bien avant la construction des grands centres Forum et Dolce Vita qui vinrent entraîner son déclin à la fin des années 1990. Le CCA est aujourd'hui déserté aussi bien par les commerçants que par les usagers, et offre des loyers très bas, fort attractifs pour des entrepreneurs dotés de petits moyens.

Le CCA arrive pour la première fois dans notre projet par la voix d'une participante australienne, missionnaire d'une église baptiste dont le siège se situe au $4^{\text {ème }}$ étage de ce centre commercial. Voici le résumé des textes enregistrés.

Le 25 octobre, Caméra 2, clip 140, minute 27 environ. Louise ${ }^{12}$ (missionnaire australienne d'une Église baptiste) parle de la fête annuelle hindoue qui a lieu au CCA, là où se trouve son église. Le sujet fait irruption à la suite du récit d'Abdurafik, à propos de la fête des musulmans à la même date. L'extrait de son intervention ce jour-là nous renseigne sur le Nouvel An hindou, sur la nourriture, les danses, le nombre de participants Hindous (« une trentaine ») aux festivités. C'est juste devant son église baptiste que ce groupe se retrouve, au $4^{\text {ème }}$ étage, dans un magasin d'articles indiens, encens et vêtements, à côté du «café des Bangladeshis où l'on peut manger des momos (sorte de raviolis népalais) ». Elle termine en se réjouissant de la diversité de populations qu'offre ce lieu.

Le 26 octobre Caméra 2, clip 087, minute 18 environ. Louise, faisant un bilan de la journée de la veille, dit qu'elle a mangé de la nourriture indienne, des momos à 2 euros à l'Avenida. Abdurafik dit qu'il connaît l'endroit. Monsieur Arsénio demande où cela se trouve

12 Tous les collaborateurs du projet ont autorisé la citation de leur nom. Ce sont des collaborateurs, pas des cobayes. 
et se souvient qu'il y a acheté son téléphone portable. Louise revient sur la réclame du momo à 2 euros.

Caméra 1, clip 85, minute 5 environ. Lucy raconte son histoire. Elle dit qu'elle a eu beaucoup de mal à trouver une place de coiffeuse et qu'après avoir terminé une formation d'esthétique $^{13}$, a ouvert un salon à l'Avenida. Clara et Elsa notent le thème de l'Avenida, Louise demande à quel étage, Clara demande si elle et Louise se sont déjà vues, et Alda regarde Lucy lorsqu'elle parle de l'Avenida. Moment crucial.

Le 27 octobre Caméra 2, clip 90, minute 23 environ. Alda à propos de sa situation financière est invitée par Elsa à parler de la condition de son mari. Elle raconte qu'elle le voit quitter la maison tous les jours mais nous fait comprendre qu'elle ne sait pas très bien ce qu'il fait. Elle ajoute qu'il possède un bureau à l'Avenida. Louise insiste pour connaître l'étage, Elsa dit à Clara que le sujet de l'Avenida doit être intégré au film.

Caméra 2, clip 99, minute 13 environ. Maria da Penha, en racontant son histoire, nous dit que le plus difficile à gérer au Portugal, ce sont ses cheveux parce qu'il n'y a aucun salon de coiffure où l'on sait s'en occuper. Elle raconte qu'elle est allée à l'Avenida, à la recherche d'un coiffeur afro qui lui avait été recommandé et elle parle de sa rencontre avec Lucy, au quatrième étage du centre commercial. Lucy étoffe l'histoire. Ce sont deux récits d'un même événement.

Le 23 mars 2013 Caméra 1, clip 09, minute 30 environ. Socorro dit qu'il y a un endroit où il y a des avocats pour les immigrants, à la suite de ce que disait Flávia à propos de la méconnaissance de la loi sur l'immigration. Socorro raconte qu'elle a vu à la Junta de Freguesia $^{14}$ de Olivais qu'il existait un cabinet d'avocats pour les immigrants au shopping Avenida. Elsa demande où (en voulant savoir à quel niveau) et Socorro parle de l'avenue Sa da Bandeira mais dit qu'elle ne sait pas à quel étage.

Chacun de ces extraits est un moment de construction involontaire du CCA en tant que thème à prendre en considération dans le cadre du projet. Le CCA est un lieu de référence pour ces personnes qui va au-delà de l'expérience personnelle de chacun. Une analyse plus détaillée des récits, que nous avons ici résumés, offre davantage d'éléments significatifs de ce moment de co-construction (analyse linguistique, interactionnelle).

Partant de cette évidence, appelons-la ainsi, le CCA est devenu un thème de dialogue interdisciplinaire avec nos collègues architectes du Centre d'Etudes Sociales. Étant professeurs

\footnotetext{
13 En fait, un CAP de coiffure.

${ }^{14}$ Échelon de l'organisation municipale portugaise, sorte de mairie de quartier.
} 
de licence et de second cycle en architecture, ils ont été séduits par l'idée de faire participer l'équipe et les volontaires liés au projet à une action commune auprès des étudiants. C'est ainsi qu'est née l'idée d'organiser un cours de formation autour du CCA, dans une logique de dialogue et de recherche interdisciplinaire entre tous les intéressés (la présentation et le programme du cours sont ci-annexés).

Auparavant déjà, le CCA était devenu un catalyseur thématique pour le film documentaire que nous avons prévu de réaliser en collaboration avec les participants des ateliers.

Nous irons donc filmer au CCA, avec l'équipe technique de la chaine télévisée de l'université de Coimbra, guidés par les participants qui ont apporté au projet cet espace architectural et urbanistique. L'idée est de suivre leurs schémas mentaux et leur expérience de ce lieu, en les superposant à un jeu de dialogue qui fait vivre l'espace : les momos népalais (nourriture, odeurs, culture), le salon de coiffure afro (corps, textures, ségrégation raciale), les téléphones portables (communication), le bureau du mari (travail), le café des bangladeshis (communauté d'appartenance, identité ethnique et nationale), l'église baptiste (religion), les avocats des immigrants (la loi).

Le scénario sera élaboré en groupe, avec le concours de tous les participants, après la séance de photos et d'images à l'Avenida. Nous avons également l'intention d'inclure dans le film des extraits de vidéos biographiques faites par les volontaires qui y participent. Ce défi a été lancé dès le début des contacts entre l'équipe de recherche de départ et les volontaires qui ont adhéré à la proposition. En avril dernier, nous avons réuni les trois groupes des premiers ateliers, en un cercle élargi, pour débattre au sujet du film. Nous avons suggéré que chaque participant réfléchisse à «son film» mais aussi qu'il apporte au projet des enregistrements autobiographiques filmés. Nous avons saisi la volonté enthousiaste dont tous ont fait preuve, mais aussi une certaine passivité et inertie, si l'on tient compte du peu de matériel reçu à ce jour. Il s'agit d'une collaboration dont nous seuls avons eu l'initiative et qui n'émane pas d'une quelconque institution ou communauté. C'est pourquoi nous ne nous dissimulons pas le poids décisif de nos propositions dans l'expérience participative. Nous affirmons cependant l'impérieuse nécessité de la participation des volontaires à la production d'une connaissance équitable, en accord avec la proposition d'agir en connaissance avec les sujets sur le terrain. Sans eux et sans elles ce travail ne saurait exister.

Il nous reste toute une année pour faire aboutir le projet mais nous avons demandé à tous les participants un bilan de l'expérience que furent les ateliers biographiques et une évaluation du travail effectué. Tous soulignent l'importance du format, du dialogue, de l'écoute et la 
nécessité de faire connaître les expériences réelles de ceux qui vivent l'immigration. En ce sens, plus qu'un moyen de donner voix aux immigrants, le projet de construire un travail en collaboration avec Abdurafik, Alda, Arsénio, Bernardino, Cristina, Dália, Elisabete, Flávia, Kouassi, Lluba, Lucy, Maria da Penha, Reginaldo, Rosana, Rosantina, Shaknoza, Socorro, Viktoria, Virgílio, traduit surtout la volonté de contribuer à la connaissance de l'immigration, de ses problèmes et de son potentiel, en une action commune d'interconnaissance et d'apprentissage. Pour ce faire, le travail des ateliers biographiques est décisif, tout comme le sont les supports audiovisuels qui doivent être utilisés dans les produits hypermédia et dans le film documentaire. Nous réaliserons également une exposition au Centre commercial Avenida, à la suite du cours d'été. Nous y exposerons les portraits en grand format des usagers de cet espace, nous afficherons les textes de leurs récits sur de vastes toiles, nous montrerons les vidéos où ils s'expriment et ferons connaître toute autre contribution, à propos du CCA.

\section{Une note de conclusion : pour tout ce qui n'a pas été dit...}

Ce texte est le point de départ d'un encadrement de l'expérience concrète de travail participatif avec des immigrants que nous développons au sein du Centre d'Etudes Sociales de l'Université de Coimbra. Il s'agit d'une première confrontation entre le cadre théorique de l'investigationaction et de la recherche partagée - telles que nos références les définissent - et notre projet. Pour cela, nous avons revisité les fondements de cette littérature scientifique, laquelle est aussi, à notre avis, une littérature civique qui établit les axes d'une économie humaine et même d'une morale sociale en les fondant sur la notion de citoyenneté participative.

Nous avons indiqué les premiers éléments permettant de faire connaître le déroulement de ce travail. Au moment d'achever cette contribution, nous avons le sentiment et la certitude qu'il resterait encore beaucoup à dire : sur l'investissement du terrain, l'établissement des relations, les très nombreuses rencontres formelles et informelles avec les personnes concernées, le maintien des contacts au fil du temps, le travail dans les ateliers, les enregistrements sonores et visuels, la préparation du tournage du film. Le cadre d'un article ne suffit pas à rendre compte de tout le matériel accumulé et des analyses multiples qu'il appelle. D'autres moyens demanderaient également à être convoqués: l'archive sonore, la photographie, la vidéo, le cinéma ; et d'autres initiatives engagées, d'autres collaborations nouées avec des collègues et des institutions du Portugal et d'autres pays. Reste que notre intention et notre volonté dans cet article comme dans le projet que nous menons vont à 
approfondir le dialogue et la connaissance, tant théorique que pratique, de la recherche participative.

\section{Références bibliographiques}

Bairon, S \& Lazaneo C. (2012). «Produção partilhada do conhecimento : do filme ao hipermédia". XXXV Congresso Brasileiro de Ciências da Comunicação. Fortaleza, CE, 3-7 Setembro 2012.

Boal, A. (1975). Teatro do oprimido e outras poéticas políticas. Rio de Janeiro : Civilização Brasileira.

Bouman, O. (2008). "Unsolicited Architecture". A collaborative project by Archis + AMO + C-LAB, Studio for Unsolicited Architecture, Design, and E-Culture, the Netherlands Architecture Institute.

Carr, W. (2013). Education, Democraty and Action Research. «Collaborative Action Research Network » (CARN). Tromso, Norvège, 7-9 nov. 2013.

Chevalier, J \& Buckles, D. J. (2013). Participatory Action Research, Theory and Methods for Engaged Inquiry. Routledge.

Dewey, J. (1938). Experience and Education. Kappa Delta.

Ferreira de Almeida, J. (2001). Em defesa da Investigação-acção. Sociologia Problemas e Práticas, 37, 175-176.

Jasanoff, S. (2004a). Science and Citizenship. Science and Public Policy, 31, 90-94.

Jasanoff, S. (2004b). States of Knowledge: The Co-production of Science and Social Order. London, UK: Routledge.

Lechner, E. (2012). Oficinas de trabalho biográfico: pesquisa, pedagogia e ecologia de saberes. Educação \& Realidade, 37/1, 71-87.

Lechner, E. \& Paulo Providência, J. (2013) Apresentação e programa do Curso de Formação Avançada «Espaços e Transições : partilhando biografias e projectos na reinvenção do Centro Comercial Avenida ». Centro de Estudos Sociais, Coimbra.

Lewin, K. (1946). Action research and minority problems. Journal of Sociological Issues, 2(4), 34-46.

Temple, Dominique (2003) Teoría de la Reciprocidad. T. I : La reciprocidad y el nacimiento de los valores humanos. T. II: La economía de reciprocidad. La Paz: PADEP/ GTZ. 